\title{
Post-HDX Deglycosylation of Fc gamma Receptor Illa Glycoprotein Enables HDX Characterization of its Binding Interface with IgG1
}

Nicole D. Wagner ${ }^{\dagger}$, Yining Huang ${ }^{\star \ddagger}$, Tun Liu^,§, Michael L. Gross ${ }^{\star \dagger}$

tDepartment of Chemistry, Washington University in St. Louis, St. Louis, MO 63130 United States

†Lilly Research Laboratories, Eli Lilly and Company, Indianapolis, IN 46285 United States

§Current affiliation: Janssen Research \& Development, Spring House, PA 19477 United States

*Corresponding authors: yining@lilly.com; mgross@wustl.edu

\section{Table of Contents}

Experimental Details

2

Figure S1. Coverage maps for 5-min incubation with pepsin at different incubation temperatures...................4

Figure S2. Coverage maps for 10-min incubation with pepsin at different incubation temperatures.................5

Figure S3. Coverage maps for 15-min incubation with pepsin at different incubation temperatures.................6

Figure S4. CD16a digestion efficiency using varied pepsin incubation times and temperatures.......................7

Figure S5. Coverage map showing all peptides identified from all PNGase A incubation conditions.................8

Figure S6. PNGase A deglycosylation products as a function of incubation time and [PNGase A] ..................8

Figure S7-1. All kinetic plots for HDX of CD16a binding IgG1 with post-HDX deglycosylation, part $1 \ldots \ldots \ldots \ldots . . . .9$

Figure S7-2. All kinetic plots for HDX of CD16a binding lgG1 with post-HDX deglycosylation, part 2. ............10

All the raw HDX data are also part of the SI as separate files 


\section{Experimental Details.}

Materials. All chemicals, pepsin beads, solvents, and the glycosidase were purchased from Sigma-Aldrich (St. Louis, MO) unless otherwise indicated and used without further purification. Deuterium oxide was purchased from Cambridge Isotope Laboratories, Inc. (Andover, MA). Human CD16a (Sino Biological Inc., Wayne, PA) and IgG1 (InvivoGen, San Diego, CA) were reconstituted in phosphate-buffered saline solution (PBS, pH 7.4) and buffer exchanged for sample clean-up using Vivaspin 500 centrifugal filters (5k MWCO, Sartorius, Goettingen, Germany). The samples were then flash frozen in liquid nitrogen and stored at $-80{ }^{\circ} \mathrm{C}$ for future analysis. CD16a and IgG1 were mixed in a molar ratio of 1.2:1 CD16a:IgG1 for bound states and incubated for at least $1 \mathrm{~h}$. Pepsin-coupled agarose beads used for in-solution digestion were buffer exchanged (Costar Spin-X $0.22 \mu \mathrm{m}$ cellulose acetate filters, Corning Life Sciences, Corning, NY) into the PNGase A compatible citric acid buffer $(100 \mathrm{mM}, \mathrm{pH} 2.2)$. An aliquot for the fully deuterated CD16a control was incubated in $1 \times \mathrm{PBS}(\mathrm{pD} 7.4)$ in $90 \%$ $\mathrm{v} / \mathrm{v} \mathrm{D}_{2} \mathrm{O} / \mathrm{H}_{2} \mathrm{O}$ for $6 \mathrm{~h}$ at $60^{\circ} \mathrm{C}, 6 \mathrm{~h}$ at room temperature, flash frozen in liquid nitrogen, and then stored at $-80{ }^{\circ} \mathrm{C}$ for use in back-exchange characterizations.

HDX Incubation, Quench, and Sample Manipulations. HDX was initiated by the addition of $95 \mu \mathrm{L}$ of $1 \times$ PBS in $\mathrm{D}_{2} \mathrm{O}(\mathrm{pD} 7.4)$ to $5 \mu \mathrm{L}$ of the unbound or bound protein sample $\left(90 \% \mathrm{v} / \mathrm{v} \mathrm{D}_{2} \mathrm{O} / \mathrm{H}_{2} \mathrm{O}\right)$, and the sample incubated on ice for $10,30,60,120,600,900,3600,7200$, or $14400 \mathrm{~s}$. The HDX reaction was quenched via a 2:3 dilution with $100 \mu \mathrm{L}$ of pepsin-coupled bead slurry in a solution of $100 \mathrm{mM}$ citric acid buffer $(\mathrm{pH} 2.2)$ and with $50 \mu \mathrm{L}$ of $1 \mathrm{M}$ TCEP in $1 \times$ PBS ( $\mathrm{pH} 2.2$ ); the final measured $\mathrm{pH}$ was 2.5. The sample was then incubated at a set temperature and time (discussed below) with a glass bead and $300 \mathrm{rpm}$ agitation to keep pepsin beads suspended (attempts without agitation resulted in very little digestion). Pepsin beads were removed from the solution by using 0.22 $\mu \mathrm{m}$ centrifugal filters (Costar Spin-X Centrifuge tube filters, cellulose acetate membrane, Corning Life Sciences, Corning, NY); the filtered solution was then incubated at a set temperature and time (discussed below). Immediately after PNGase A incubation, the sample was flash frozen in liquid nitrogen and stored at $-80{ }^{\circ} \mathrm{C}$ for later analysis.

Pepsin Digestion Optimization. Peptide products of CD16a without deuteration were mapped using variable pepsin incubation times and temperatures $\left(5,10\right.$, and $15 \mathrm{~min}$ at 7,11 , and $15^{\circ} \mathrm{C}$ ) to maximize pepsin digestion and peptide recovery. All samples were characterized using LC/MSMS, and the data processed using Byonic ${ }^{\mathrm{TM}}$ and compiled by using Byologic $®$ (version 2.9-59, Protein Metrics Inc., Cupertino, CA). Coverage maps are shown in Figures S1-S3.

The analysis was repeated with "fully deuterated" CD16a to characterize the back-exchange extent observed for each condition. The number of identified peptides, the relative abundance of identified peptides after searching with a cumulative in-silico list, and the $\% \mathrm{H} / \mathrm{D}$ back-exchange are summarized for each of the conditions in Figure S4. There were large gains in peptide identifications and peptide abundances when the temperature was increased from $7{ }^{\circ} \mathrm{C}$ to $11^{\circ} \mathrm{C}$, but only small gains when the temperature was increased to $15^{\circ} \mathrm{C}$. Similarly, a large benefit in peptide production was observed when the incubation time was increased from 5 to 10 min, but further increase to 15-minutes yielded minimal improvement. Thus, the conditions of pepsin-coupled bead incubation for $10-\min$ at $11^{\circ} \mathrm{C}$ was chosen for all further experiments.

PNGase A Deglycosylation Optimization. By using the chosen pepsin-coupled bead incubation time and temperature $\left(11^{\circ} \mathrm{C}\right.$ for 10 minutes), the effect of PNGase $A$ concentration and incubation time were characterized. Peptide products of CD16a without deuteration were incubated at $11^{\circ} \mathrm{C}$ with $2 \mu \mathrm{U} / \mathrm{pmol}(\mathrm{E} / \mathrm{S})$ and $6.25 \mu \mathrm{U} / \mathrm{pmol}$ of PNGase A for 2, 4 and 6-min. The resulting peptides were characterized by using LC/MSMS, and the data processed by using Byonic ${ }^{\mathrm{TM}}$ and compiled using Byologic $®$ (version 2.9-59, Protein Metrics Inc., Cupertino, CA). A coverage map showing all identified peptides is shown in Figure S5, where deglycosylation is observed as an apparent $N$ to $D$ mutation. The deglycosylated peptides identified by using each of the conditions were variable and unpredictable, potentially owing to data-dependent MSMS not choosing the deglycosylated peptides; however, searching with an in-silico list identified all deglycosylated peptides by using each of the conditions with $<5 \mathrm{ppm}$ error. The relative abundances of deglycosylated peptides for each of the conditions are shown in Figure S6. The use of $6.25 \mu \mathrm{U} / \mathrm{pmol}$ of PNGase A had a marked effect on the deglycosylated peptide yield as characterized by LC/MSMS. Increasing the incubation time from 2 to 4 min also increased the deglycosylated peptide yield, whereas increasing the incubation time from 4 to 6-min had almost no observed effect. Consequently, we used 4-min incubation with $6.25 \mu \mathrm{U} / \mathrm{pmol}$ of PNGase A for HDX-MS analysis of CD16a binding with lgG1. These optimized pepsin and PNGase A incubation conditions enabled an 
LCMS analysis of the "fully deuterated" CD16a to examine the back-exchange extent. The average backexchange was $\sim 54 \%$ (median $53 \%$, range $39-80 \%$ ) as calculated below. ${ }^{1}$

Jensen et al. suggested that deglycosylation efficiency may be affected by peptide length; however, no such correlation was observed (Figure S6). ${ }^{2}$ Instead, we observed a clear dependence on glycosylation site. The identity of the glycan at each of the sites is reportedly variable. ${ }^{3}$ This variation in glycan identities may also be responsible for inefficient deglycosylation at the different glycosites.

Mass Spectrometric Analysis. All mass spectral analyses (LC/MS and LC/MSMS) were performed using a custom-built liquid chromatography (LC) assembly incorporating trapping and desalting on a ZORBAX Eclipse XDB C8 column (2.1 mm x $15 \mathrm{~mm}$, Agilent, Santa Clara, CA) and analytical separation with a Hypersil Gold C18 column (2.1 mm x $50 \mathrm{~mm}$, Thermo Fisher, Waltham, MA). All valves, tubes, and columns (except for the pepsin column, which suffered a loss in activity at low temperature) were submerged in an ice-water bath to minimize back-exchange. The peptides were then introduced via electrospray ionization into an LTQ-FTICR mass spectrometer (Thermo Fisher, Waltham, MA). The LC-MS/MS results from mapping were searched by using Byonic $^{\mathrm{TM}}$ and compiled using Byologic ${ }^{\circledR}$ (version 2.9-59, Protein Metrics Inc., Cupertino, CA).

HDX data processing. Data analysis of continuous HDX was carried out using HDExaminer (version 2.5.0, Sierra Analytics, Inc., Modesto, CA). Back-exchange was calculated as follows: ${ }^{1}$

$$
\% \text { Back-exchange }=\left(1-\frac{D_{\text {rep }}}{N \times D_{\text {frac }}}\right) \times 100
$$

where $D_{\text {rep }}$ is the reported deuterium uptake for the peptide (from HDExaminer relative to the non-deuterated, 0 $\mathrm{s}$, isotope distribution), $\mathrm{N}$ is the theoretical number of exchangeable backbone amides (where prolines and the $\mathrm{N}$-terminal residue of the peptide are not considered), and $\mathrm{D}_{\text {frac }}$ is the fraction of $\mathrm{D}$ in the exchange buffer ( 0.9 in this case).

The cumulative differences in HDX (Figure 3A) were calculated as the sum of all differences at all time points in $\mathrm{Da}$ :

$$
\text { Summed } \Delta H X=\sum\left(D_{t, \text { bound }}\right)-\sum\left(D_{t, \text { unbound }}\right)
$$

where $D_{t}$ is the deuterium uptake in $D a$ at each time point for the respective state (unbound or bound).

The global significance limit (Figure 3A) was calculated as previously discussed. ${ }^{4,5}$ Briefly, the pooled standard deviation was calculated for the entire dataset for each state:

$$
s_{\text {pooled }}=\sqrt{\frac{\sum s^{2}}{N}}
$$

where $s$ is all standard deviations across the dataset and $N$ is the number of data points (the number of timepoints * the number of peptides - the number of data points for which a standard deviation was not possible). From the pooled standard deviations of both states, the standard error of the mean (SEM) was calculated:

$$
S E M_{\triangle \overline{H X}}=\sqrt{\frac{s_{p, \text { unbound }}^{2}}{n_{\text {unbound }}}+\frac{s_{p, \text { bound }}^{2}}{n_{\text {bound }}}}
$$

where $\mathrm{n}$ is the number of experimental technical replicates (in this case 2 for both). Then, using the SEM and the t-value for a two-tailed Student's t-distribution, $p<0.02$ and 2 degrees of freedom (for duplicates of two states, $2+2$ - 2), we calculated the confidence interval or global $\Delta \mathrm{HX}$ significance limit:

$$
\pm C I_{\Delta \overline{H X}}=t \times S E M_{\Delta \overline{H X}}
$$

The calculated significance limit for $p<0.02$ of this dataset is $0.77 \mathrm{Da}$.

\section{References}

1. Masson, G. R.; Burke, J. E.; Ahn, N. G.; Anand, G. S.; Borchers, C.; Brier, S.; Bou-Assaf, G. M.; Engen, J. R.; Englander, S. W.; Faber, J.; Garlish, R.; Griffin, P. R.; Gross, M. L.; Guttman, M.; Hamuro, Y.; Heck, A. J. R.; Houde, D.; lacob, R. E.; Jørgensen, T. J. D.; Kaltashov, I. A.; Klinman, J. P.; Konermann, L.; Man, P.; Mayne, L.; Pascal, B. D.; Reichmann, D.; Skehel, M.; Snijder, J.; Strutzenberg, T. S.; Underbakke, E. S.; Wagner, C.; Wales, T. E.; Walters, B. T.; Weis, D. D.; Wilson, D. J.; Wintrode, P. L.; Zhang, Z.; Zheng, J.; Schriemer, D. C.; Rand, K. D., Recommendations for performing, interpreting and reporting hydrogen deuterium exchange mass spectrometry (HDX-MS) experiments. Nature Methods 2019, 16 (7), 595-602. 
2. Jensen, P. F.; Comamala, G.; Trelle, M. B.; Madsen, J. B.; Jørgensen, T. J. D.; Rand, K. D., Removal of N-Linked Glycosylations at Acidic $\mathrm{pH}$ by PNGase A Facilitates Hydrogen/Deuterium Exchange Mass Spectrometry Analysis of N-Linked Glycoproteins. Anal. Chem. 2016, 88 (24), 12479-12488.

3. Zeck, A.; Pohlentz, G.; Schlothauer, T.; Peter-Katalinić, J.; Regula, J. T., Cell Type-Specific and Site Directed N-Glycosylation Pattern of FcyRIIla. J. Proteome Res. 2011, 10 (7), 3031-3039.

4. Houde, D.; Berkowitz, S. A.; Engen, J. R., The Utility of Hydrogen/Deuterium Exchange Mass Spectrometry in Biopharmaceutical Comparability Studies. J. Pharm. Sci. 2011, 100 (6), 2071-2086.

5. Weis, D. D., Comment on Houde, D.; Berkowitz, S. A.; Engen, J. R., The Utility of Hydrogen/Deuterium Exchange Mass Spectrometry in Biopharmaceutical Comparability Studies. J. Pharm. Sci. 2011, 100, 2071-2086. J. Pharm. Sci. 2019, 108 (2), 807-810.

LhCD16a - CD16a - haman

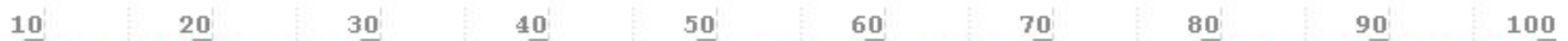

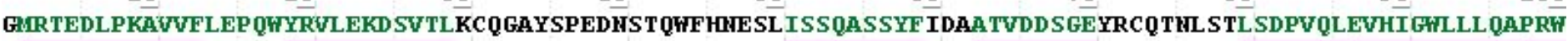

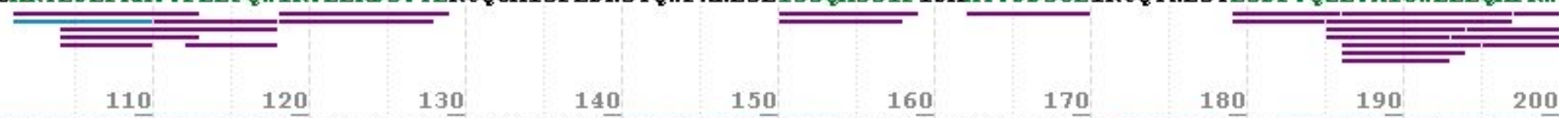

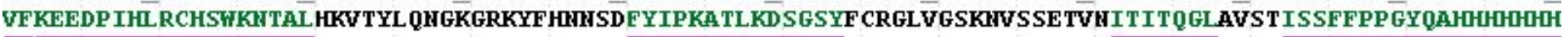
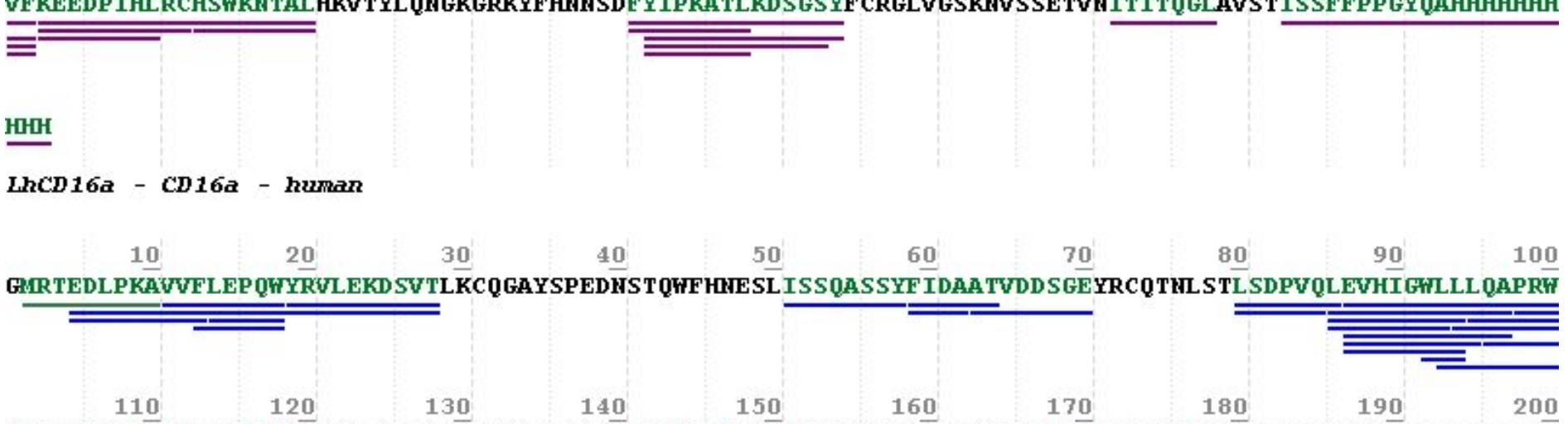

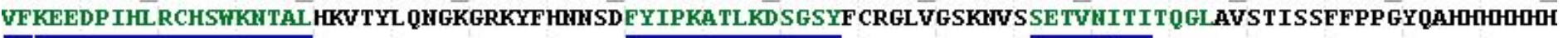

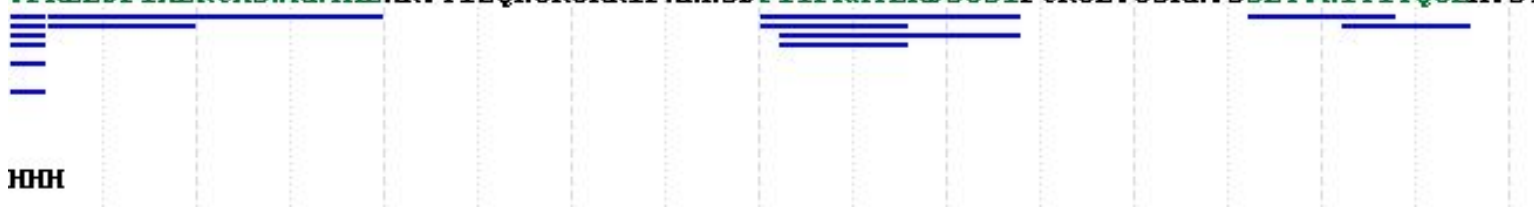

LhCD16a - CD16a - human

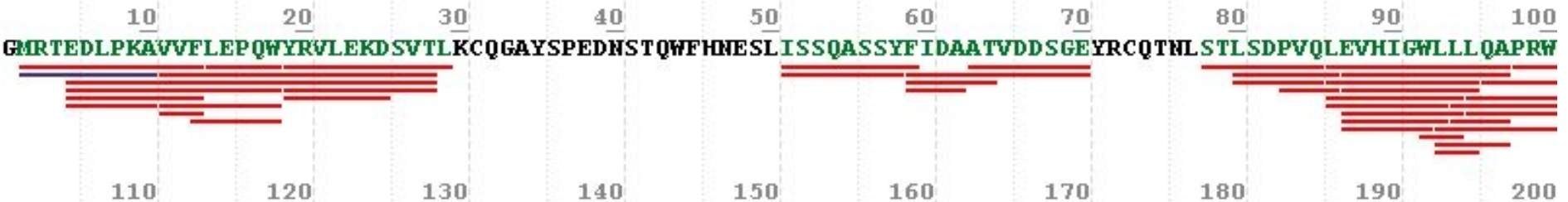

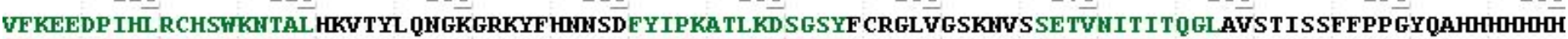

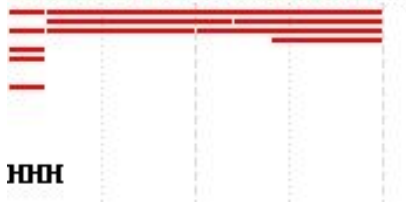

Figure S1. Coverage maps for 5-min incubation with pepsin at different incubation temperatures. Identified peptides after 5-min incubation with pepsin-coupled beads at 7,11 , and $15{ }^{\circ} \mathrm{C}$ (top to bottom and blue, purple, and red, respectively). Note the numbering is +2 from that reported elsewhere in the manuscript. 
10

40

50

60

70

$8 \underline{0}$

90

100

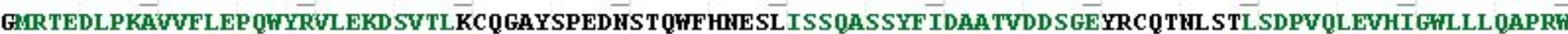

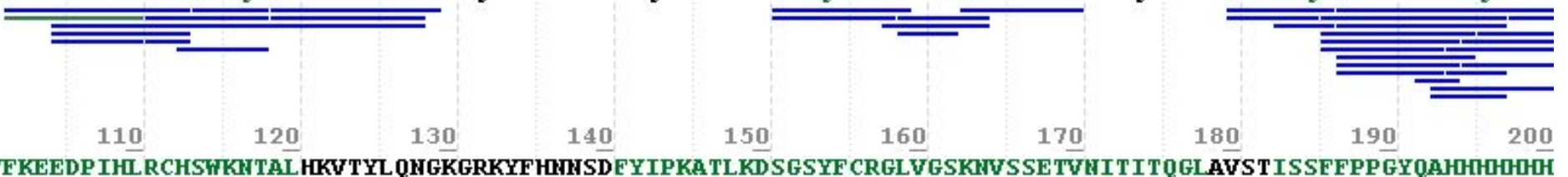

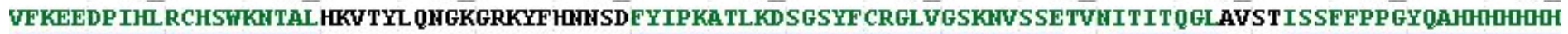
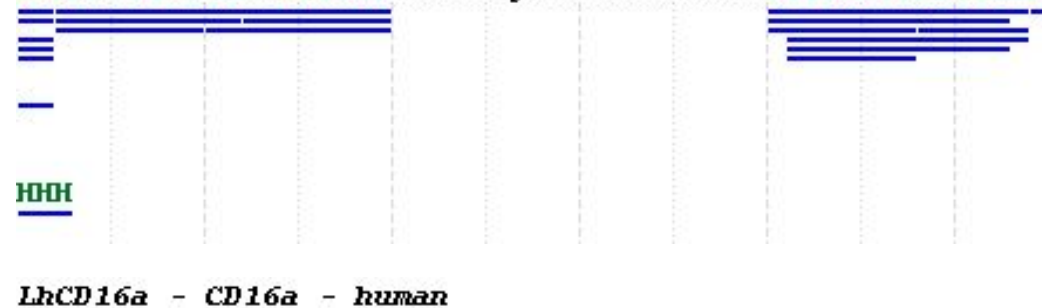

LhCD16a - CD16a - human

\begin{tabular}{lllllllll}
10 & 20 & 30 & 40 & 50 & 60 & 70 & 80 & 90 \\
\hline
\end{tabular}

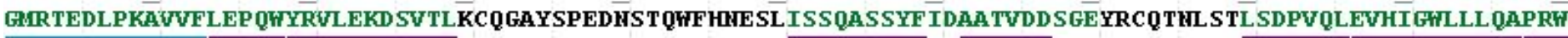

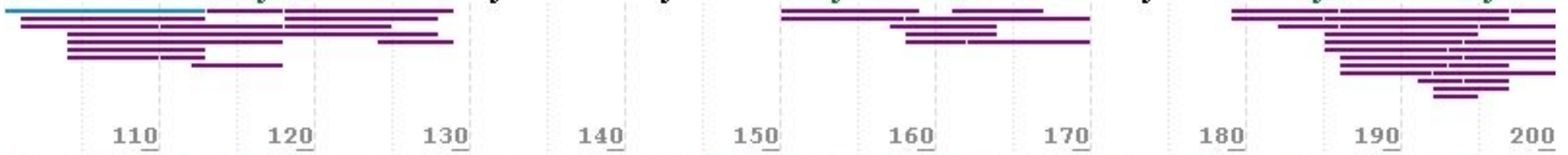

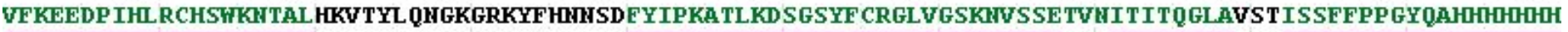

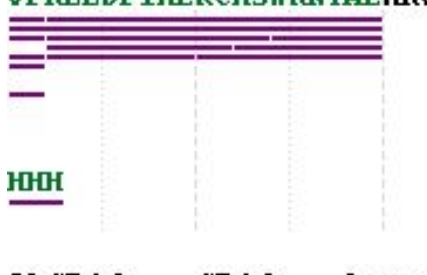

LhCD16a - CD16a - human
10
$2 \underline{0}$
$3 \underline{0}$
40
50
$6 \underline{0}$
$7 \underline{0}$
80
90
$10 \underline{0}$

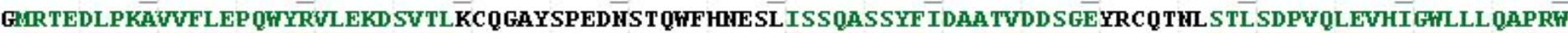

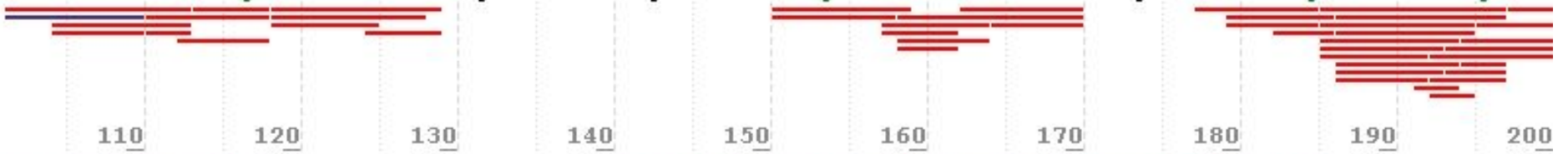

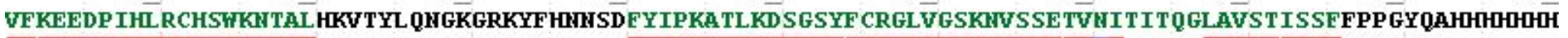
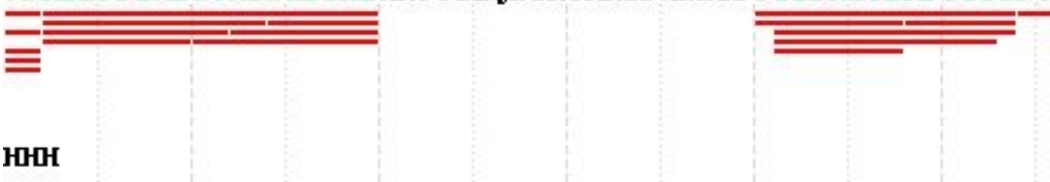

Figure S2. Coverage maps for 10-min incubation with pepsin at different incubation temperatures.

Identified peptides after 10-min incubation with pepsin-coupled beads at 7,11 , and $15{ }^{\circ} \mathrm{C}$ (top to bottom and blue, purple, and red, respectively). Note the numbering is +2 from that reported elsewhere in the manuscript. 
10

30

40

50

60

70

80

90

100

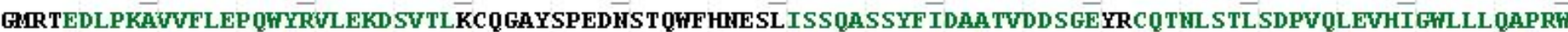

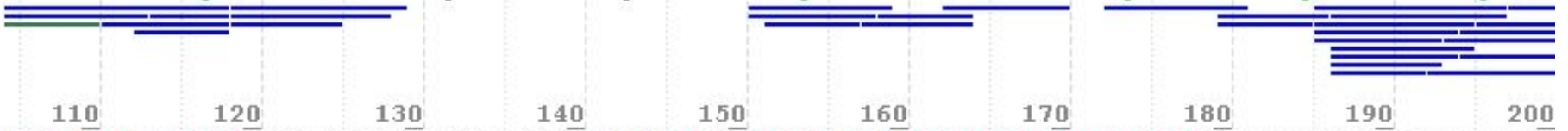

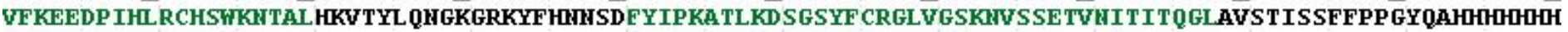
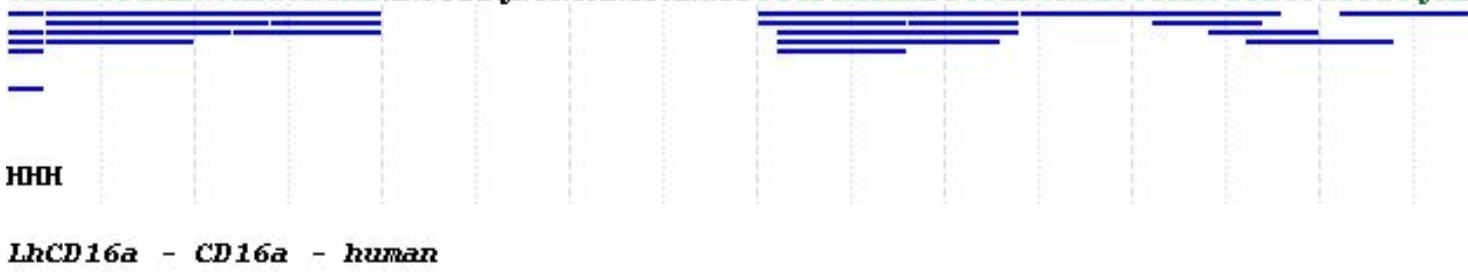

LhCD16a - CD16a - human
10
$2 \underline{0}$
30
40
$5 \underline{0}$
$6 \underline{0}$
$7 \underline{0}$
80
90
$10 \underline{0}$

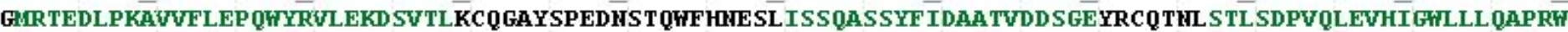

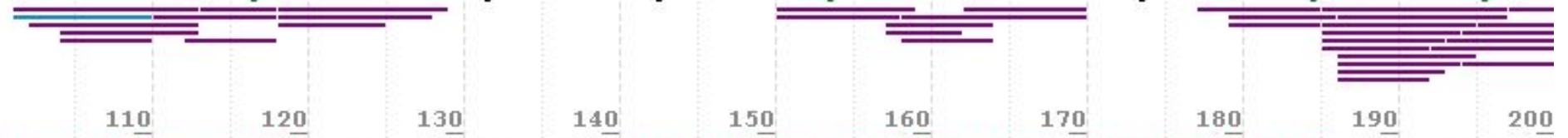

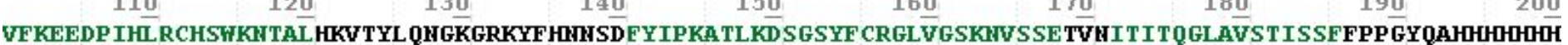
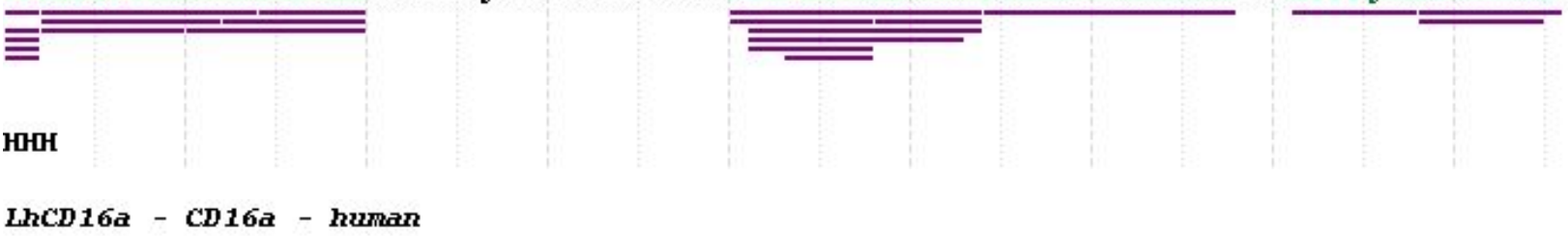

LhCD16a - CD16a - human

$30 \quad 40$

50

60

$7 \underline{0}$

$8 \underline{0} \quad 9 \underline{0}$

100

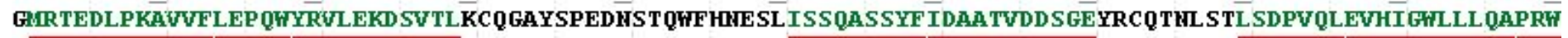

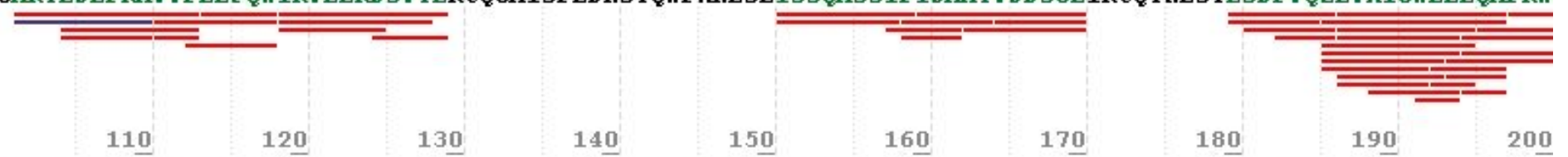

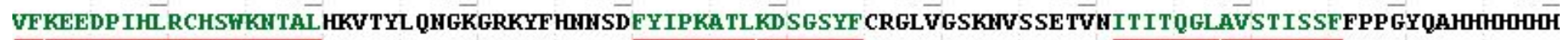
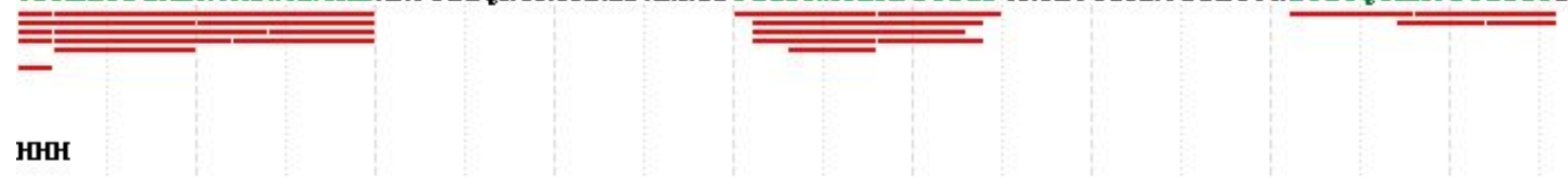

Figure S3. Coverage maps for 15-min incubation with pepsin at different incubation temperatures.

Identified peptides after 15-min incubation with pepsin-coupled beads at 7,11 , and $15{ }^{\circ} \mathrm{C}$ (top to bottom and blue, purple, and red, respectively). Note the numbering is +2 from that reported elsewhere in the manuscript. 


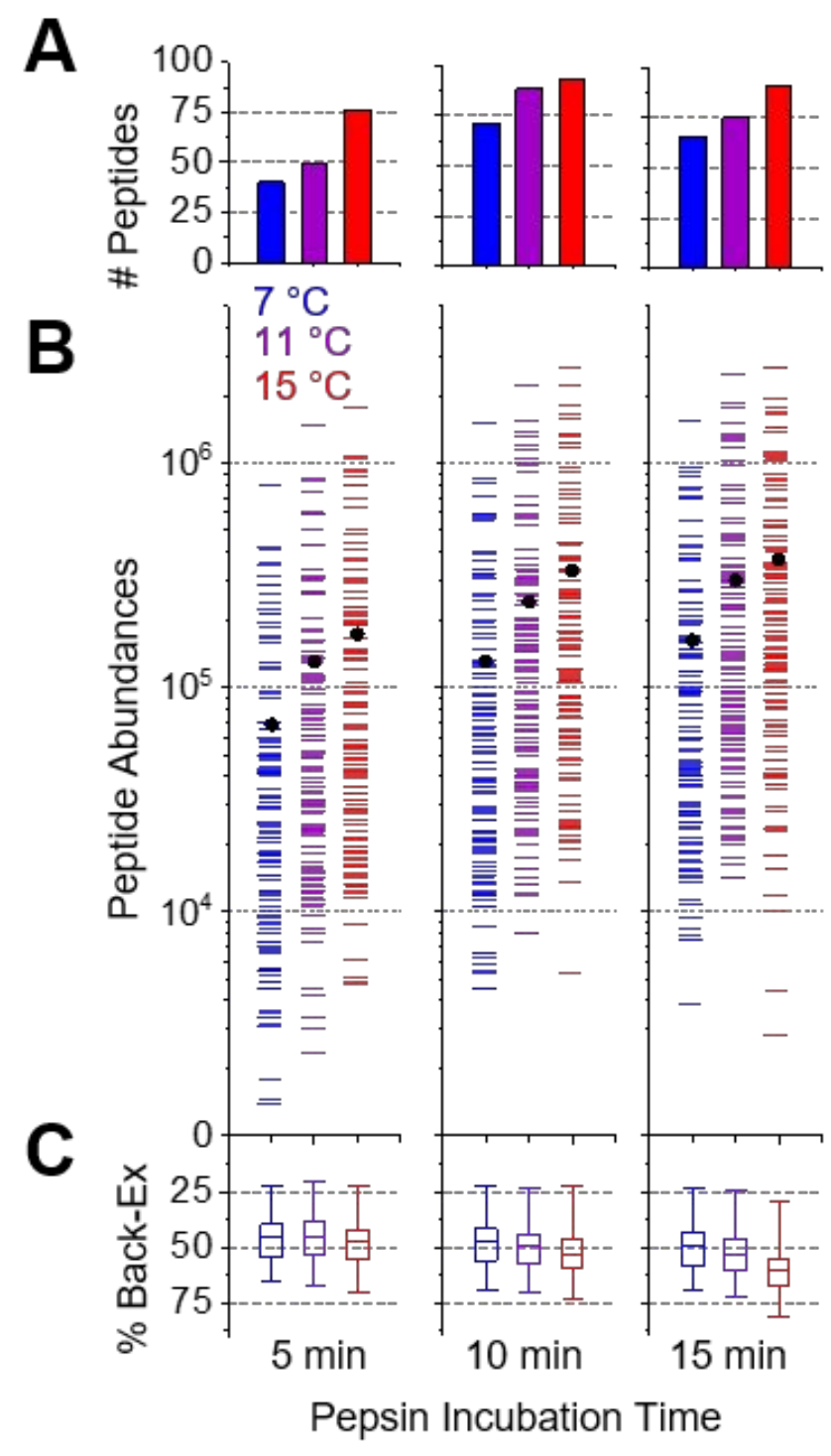

Figure S4. CD16a digestion efficiency using varied pepsin incubation times and temperatures.

(A) Count of unique peptides identified by LC-MS/MS, (B) absolute (bar) and average (circle) peptide abundances of all 113 identified peptides after in-silico search, and $(C)$ interquartile range of \% back-exchange for all observed peptides from a the hypothetically $90 \%$ deuterated protein sample after in-solution incubation with pepsin coupled beads for $5-15 \mathrm{~min}$ (left to right) at $7^{\circ} \mathrm{C}$ (blue), $11^{\circ} \mathrm{C}$ (purple), and $15^{\circ} \mathrm{C}$ (red). 
10

20

30

40

50

60

70

80

90

100

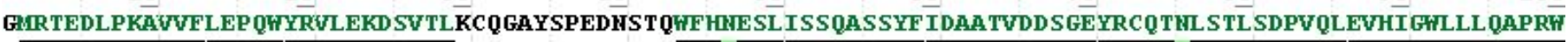

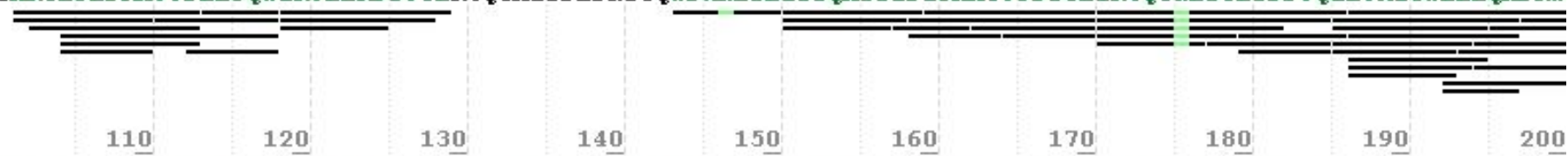

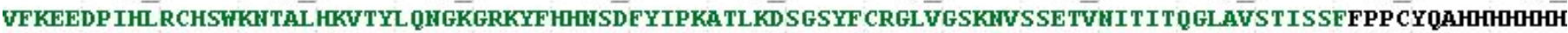

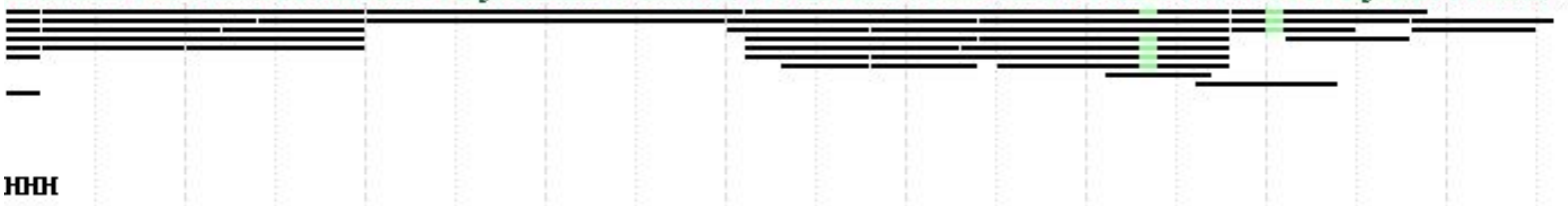

Figure S5. Coverage map showing all peptides identified from all PNGase A incubation conditions. All peptides identified after 10-minute incubation with pepsin-coupled beads at $11^{\circ} \mathrm{C}$ and PNGase $\mathrm{A}$ deglycosylation at $11^{\circ} \mathrm{C}$. Light green bars show peptides identified with an $\mathrm{N}$ to $\mathrm{D}$ apparent mutation (i.e. deglycosylation of an $\mathrm{N}$-linked glycopeptide). Note the numbering is +2 from that reported elsewhere in the manuscript.

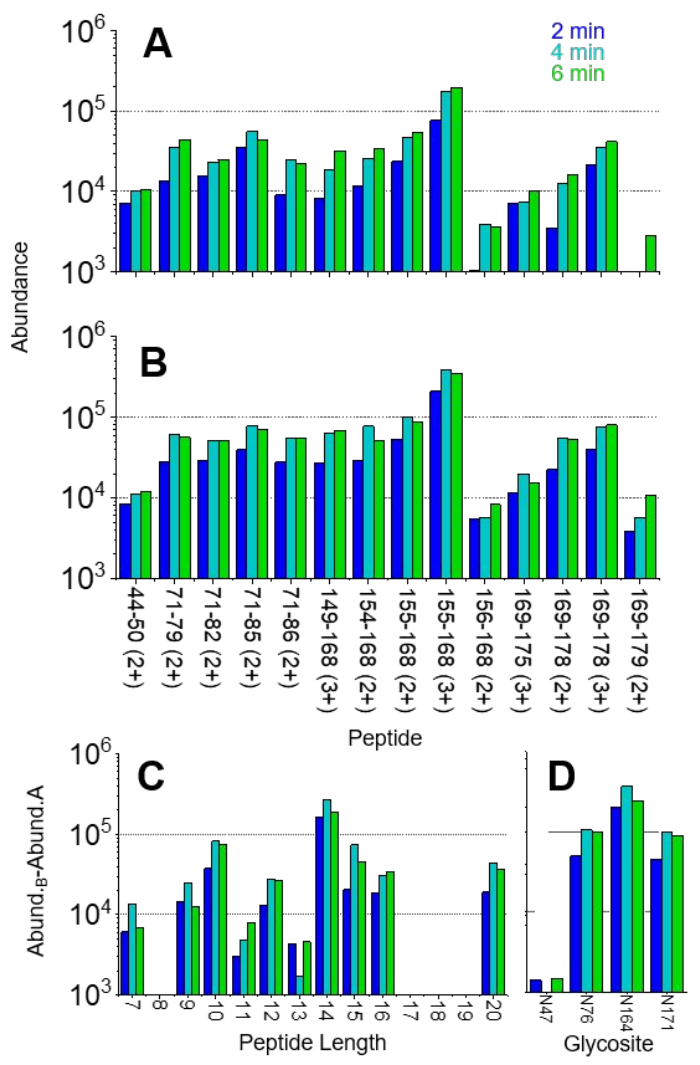

Figure S6. PNGase A deglycosylation products as a function of incubation time and [PNGase A].

(A-B) Abundances of LC-MS/MS identified deglycosylated peptides after 10-min pepsin bead incubation at 11 ${ }^{\circ} \mathrm{C}$ and $2 \mathrm{~min}$ (blue), $4 \mathrm{~min}$ (cyan), and $6 \mathrm{~min}$ (green) incubation with (A) $2 \mu \mathrm{U} / \mathrm{pmol}(\mathrm{E} / \mathrm{S}$ ) and (B) $6.25 \mu \mathrm{U} / \mathrm{pmol}$ of PNGase A. These abundances are the product of an in-silico search using a master peptide list from all LCMS/MS analyses. Difference in the abundances of observed deglycosylated peptides as a function of PNGase A concentration $(6.25 \mu \mathrm{U} / \mathrm{pmol}-2 \mu \mathrm{U} / \mathrm{pmol})$ and $(\mathrm{C})$ peptide length or $(\mathrm{D})$ glycosylation site. Note the numbering is +2 from that reported elsewhere in the manuscript. 

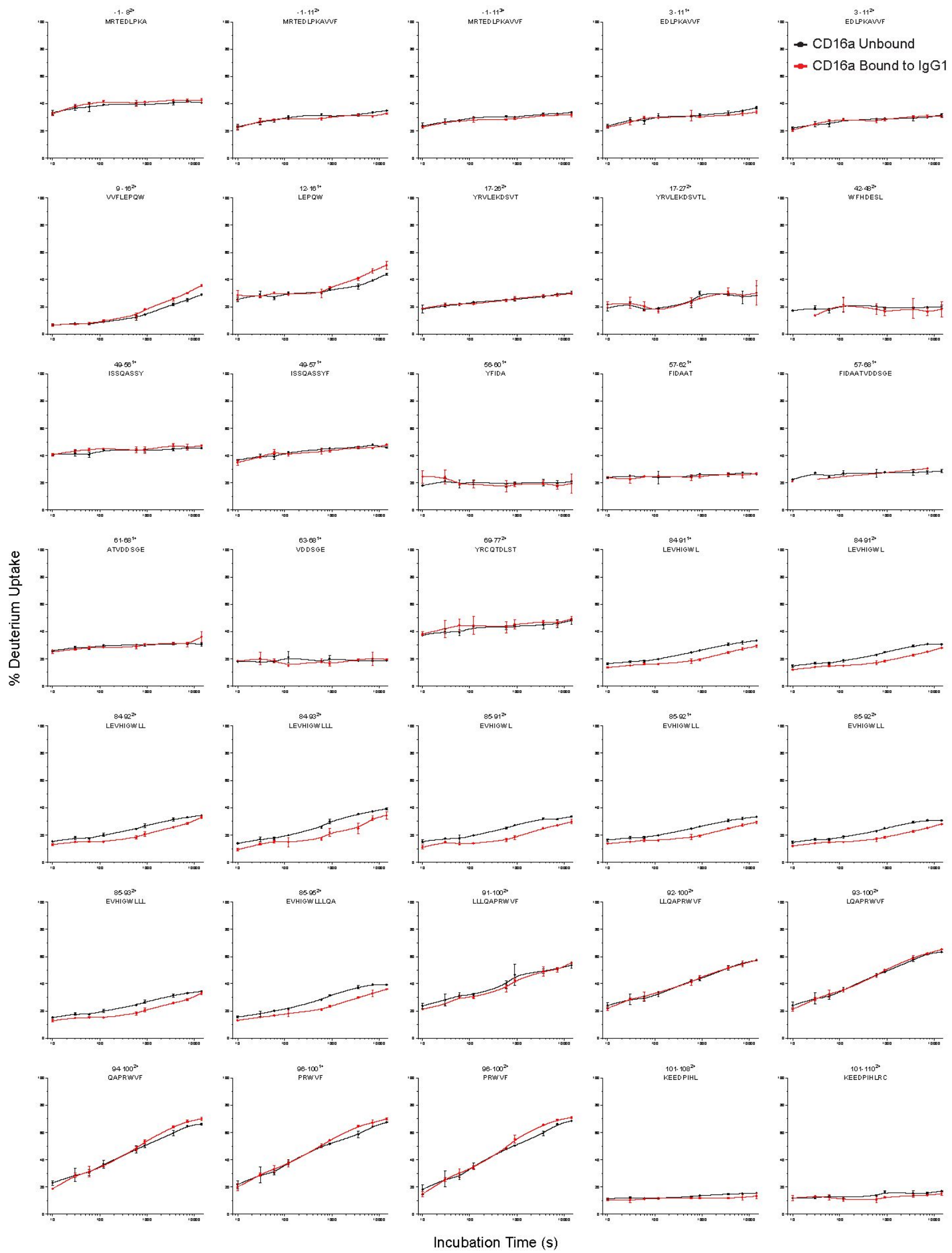

Figure S7-1. All kinetic plots for HDX of CD16a binding lgG1 with post-HDX deglycosylation, part 1. 

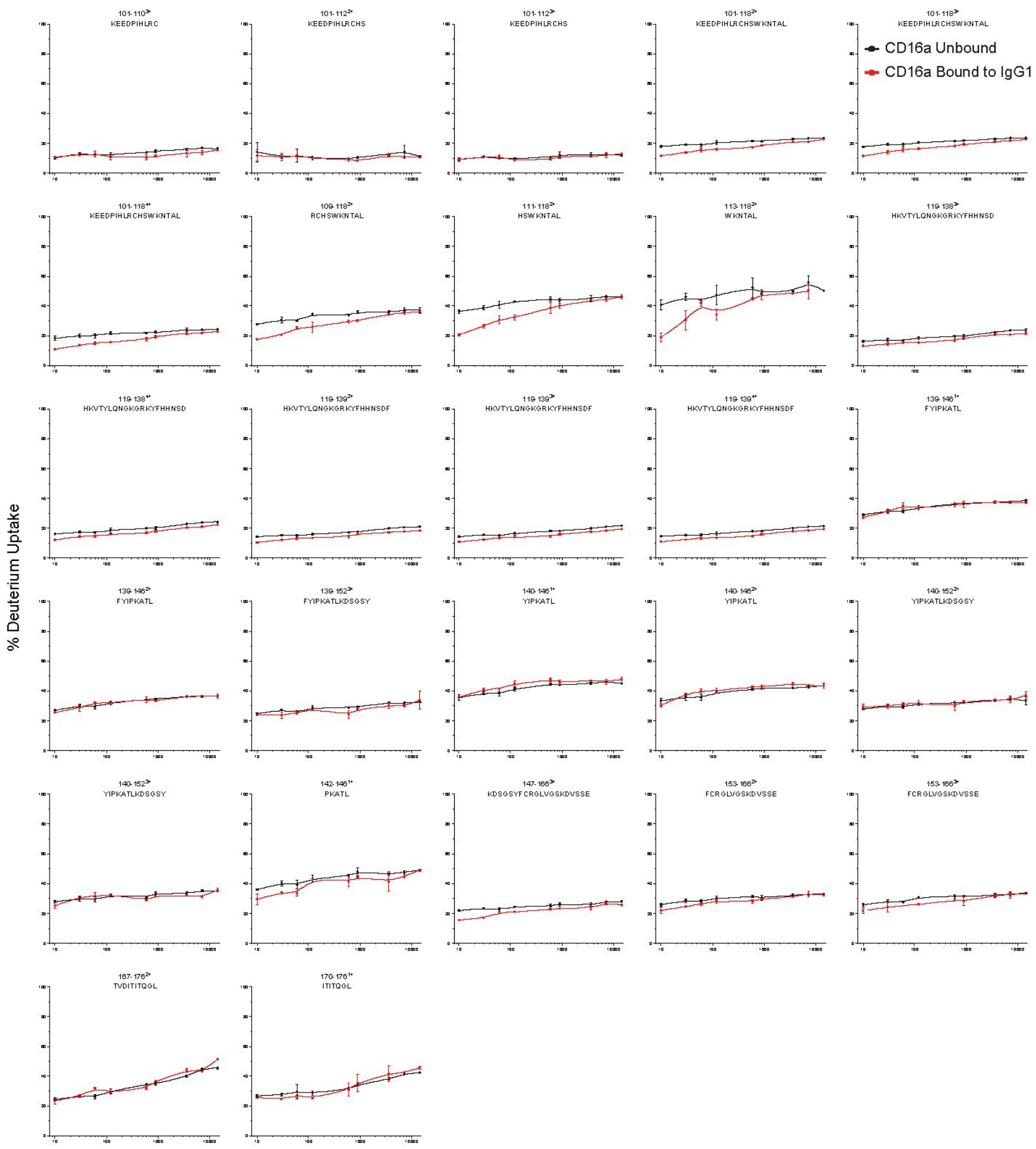

Incubation Time (s)

Figure S7-2. All kinetic plots for HDX of CD16a binding IgG1 with post-HDX deglycosylation, part 2. 